॥ This article has been updated from its originally published version to correct the affiliations. See the corresponding erratum notice, DOI: 10.3171/2019.10.JNS172148a. «

\title{
Developing an optimal follow-up strategy based on the natural history of nonfunctioning pituitary adenomas
}

\author{
*Jung Hee Kim, MD,,2,4 Yun-Sik Dho, MD,, 2,3 Yong Hwy Kim, MD,, ,3 Jung Hyun Lee, RN,, 2,3 \\ Ji Hyun Lee, MD, ${ }^{1}$ A. Ram Hong, MD, ${ }^{1}$ and Chan Soo Shin, MD, PhD ${ }^{1,2}$
}

\begin{abstract}
${ }^{1}$ Department of Internal Medicine, ${ }^{2}$ Pituitary Center, ${ }^{3}$ Department of Neurosurgery, Seoul National University Hospital, Seoul National University College of Medicine, and ${ }^{4}$ Department of Molecular Medicine and Biopharmaceutical Sciences, Graduate School of Convergence Science and Technology, Seoul National University, Seoul, Republic of Korea
\end{abstract}

\begin{abstract}
OBJECTIVE The natural history and proper algorithm for follow-up testing of nonfunctioning pituitary adenomas (PAs) are not well known, despite their relatively high prevalence. The aim of this study was to suggest the optimal follow-up algorithm for nonfunctioning PAs based on their natural history.

METHODS The authors followed up 197 patients with nonfunctioning PAs that had not been treated (including surgery and radiation therapy) at the time of detection, in a single center, between March 2000 and February 2017. They conducted a hormone test, visual field test, and MRI at the time of diagnosis and yearly thereafter.

RESULTS The overall median follow-up duration was 37 months. Microadenomas $(n=38)$ did not cause visual disturbance, pituitary apoplexy, or endocrine dysfunction. The incidence of patients with tumor volume growth $\geq 20 \%$ was higher for macroadenomas than microadenomas (13.8 vs 5.0 per 100 person-years [PYs], $p=0.002$ ). The median time to any tumor growth was 4.8 years $(95 \% \mathrm{Cl} 3.4-4.8$ years) for microadenomas and 4 years $(95 \% \mathrm{Cl} 3.3-4.2$ years) for macroadenomas. The overall incidence of worsening visual function was 0.69 per $100 \mathrm{PY}$. Patients with a tumor volume growth rate $\geq 0.88 \mathrm{~cm}^{3} / y e a r(n=20)$ had a higher incidence of worsening visual function (4.69 vs 0.30 per $100 \mathrm{PYs}, p<$ 0.001). The tumor growth rate of all microadenomas was $<0.88 \mathrm{~cm}^{3} / y e a r$. The median time to tumor growth $\geq 20 \%$ was 3.3 years $\left(95 \% \mathrm{Cl} 1.8-3.9\right.$ years) in patients with a tumor growth rate $\geq 0.88 \mathrm{~cm}^{3} /$ year and 4.9 years $(95 \% \mathrm{Cl} 4.6-7.2$ years) in patients with a tumor growth rate $<0.88 \mathrm{~cm}^{3} /$ year.
\end{abstract}

CONCLUSIONS The authors have devised a follow-up strategy based on the tumor volume growth rate as well as initial tumor volume. In patients with microadenomas, the next MRI study can be performed at 3 years. In patients with macroadenomas, the second MRI study should be performed between 6 months and 1 year to assess the tumor growth rate. In patients with a tumor growth rate $\geq 0.88 \mathrm{~cm}^{3} /$ year, the MRI study should be performed within 2 years. In patients with a tumor growth rate $<0.88 \mathrm{~cm}^{3} / y e a r$, the MRI study can be delayed until 4 years.

https://thejns.org/doi/abs/10.3171/2018.4.JNS172148

KEYWORDS follow-up; natural history; nonfunctioning; pituitary adenoma; observation; pituitary surgery

$\mathrm{W}$ ITH advances in imaging techniques, pituitary adenomas (PAs) are frequently incidentally detected, even without the presentation of symptoms. European studies have reported that the prevalence of PAs ranges from 1 of 865 to 1 of 2688 in communitydwelling adults. ${ }^{1,3,9,16,21}$ In 2013, the Korean Central Cancer Registry reported that pituitary tumors were the second most common type of brain tumor (18.0\%) among 11,827 patients diagnosed with primary brain tumors. ${ }^{13}$ In studies that included 1718 patients, $14 \%$ to $54 \%$ had clinical nonfunctioning PAs. ${ }^{1,3,9,16,21}$
Immediate medical or surgical treatment is necessary for functioning PAs, such as prolactinoma, acromegaly, and Cushing's disease. However, many nonfunctioning PAs, e.g., microadenomas, do not cause symptoms, and absolute surgical indications are mass effects, such as unremitting headaches and the development of visual field defects. ${ }^{11}$ It is also important to note that transsphenoidal surgery is accompanied by major complications, such as CSF leakage, meningitis, new hormone deficiency, and persistent diabetes insipidus in $<5 \%$ of cases and mortality in $<1 \%$ of cases. ${ }^{17}$ Therefore, the wait-and-see strategy

ABBREVIATIONS ACTH = adrenocorticotropic hormone; FSH = follicle-stimulating hormone; $\mathrm{LH}=$ luteinizing hormone; $\mathrm{PA}=$ pituitary adenoma; $\mathrm{PY}=$ person-year; $\mathrm{TSH}=$ thyroid-stimulating hormone; VIS = visual impairment scale.

SUBMITTED August 27, 2017. ACCEPTED April 5, 2018.

INCLUDE WHEN CITING Published online September 14, 2018; DOI: 10.3171/2018.4.JNS172148.

* J.H.K. and Y.S.D. contributed equally to this work. 
is usually applied in cases of asymptomatic nonfunctioning PAs.

For conservatively managed nonfunctioning PAs, controversy surrounds the proper algorithm for hormone assessment, visual field testing, and imaging as a follow-up method. This debate is attributed to a lack of evidence pertaining to the natural history of PAs, despite their relatively high prevalence. Previous longitudinal cohort studies have been limited by their small sample sizes and heterogeneity, with only 2 studies including more than 50 participants. . $^{2,-6,8,10,12,14,15,18-20}$ In addition, some studies have reported neither endocrine dysfunction nor the definition of an increase in tumor size..$^{2,4,8,18,20}$ Even the Endocrine Society's guidelines were dependent on the experience of a task force of experts. ${ }^{11}$ These guidelines recommend that, for macroadenomas, MRI should be performed at 6 months, 1 year, annually for 3 years, and less frequently thereafter, if stable. For microadenomas, MRI is recommended annually for 3 years, and less frequently thereafter, if stable. ${ }^{11}$

In this retrospective cohort study, which, to our knowledge is the largest to date in terms of sample size, we aimed to investigate the natural history of nonfunctioning PAs and propose an optimal follow-up algorithm according to the initial adenoma size and tumor growth rate.

\section{Methods \\ Study Participants}

The present study was designed as a retrospective cohort study. We identified 197 consecutive patients who were diagnosed between March 2000 and February 2017 with clinically nonfunctioning PAs at our institution. The inclusion criteria were 1) imaging features of the PA;2) no clinical or biochemical evidence of hormone excess; 3 ) follow-up without any treatment at the time of detection; and 4) 1 or more repeat MRI studies during the followup period. We excluded patients with 1) imaging features suggestive of craniopharyngioma or Rathke's cleft cyst; 2) previous surgery or radiation therapy; and 3) mass effect, such as visual field defects or pituitary apoplexy at baseline.

The follow-up duration was defined as the period from the time of diagnosis to the last MRI study or the presentation of new visual symptoms. In patients who had undergone surgery for visual impairment that occurred during the follow-up, we set the time when the last MRI study was performed before surgery as the end of followup. In the cases of apoplexy occurring during follow-up, the MRI study performed immediately after apoplexy was defined as the last MRI. The outcomes of the present study were worsening visual function, endocrine dysfunction, pituitary apoplexy, and tumor growth.

The study was approved by the ethics committee of our hospital, and written informed consent was waived due to the study's retrospective design.

\section{Hormone Function Assessment}

Endocrine function was assessed in all patients at the time of diagnosis and yearly during follow-up, except for the function of the growth hormone/insulin-like growth factor-1 axis, which was assessed less frequently. The levels of adrenocorticotropic hormone (ACTH), serum cortisol, free T4, thyroid-stimulating hormone (TSH), prolactin, luteinizing hormone $(\mathrm{LH})$, follicle-stimulating hormone (FSH), estradiol, and total testosterone were measured by radioimmunoassay and immunoradiometric assay, between 8 and $10 \mathrm{AM}$. ACTH deficiency was defined as a peak cortisol level $\leq 18 \mu \mathrm{g} / \mathrm{dl}$ after a short Synacthen test, or in cases in which dynamic testing was not available, a low morning cortisol level $(<5 \mu \mathrm{g} / \mathrm{dl})$ with a low to normal ACTH level (10-65 pg/ml). A low free T4 level $(<0.70 \mathrm{ng} / \mathrm{dl})$ and a low to normal TSH level (reference range 0.4-4.1 $\mu \mathrm{IU} / \mathrm{ml}$ ) indicated TSH deficiency. In premenopausal women, oligomenorrhea associated with estradiol levels $<50 \mathrm{pg} / \mathrm{ml}$, and normal or low FSH/LH levels indicated FSH/LH deficiency. Normal menopause was defined by FSH levels $>30 \mathrm{mIU} / \mathrm{ml}$ and estradiol levels $<50 \mathrm{pg} / \mathrm{ml}$ in women with hypopituitarism. In men, low testosterone levels and low to normal FSH/LH levels indicated central hypogonadism. New-onset worsened endocrine dysfunction was defined as a loss of $\geq 1$ of the hormonal axis during follow-up. Improved endocrine dysfunction was defined as a gain of $\geq 1$ of the hormonal axis during the follow-up.

\section{Ophthalmological Assessments}

Ophthalmological assessments consisted of visual acuity and visual field examinations. The visual field was evaluated using the Goldmann visual field test or the Humphrey visual field analyzer. Ophthalmological assessments were conducted at the time of the initial PA diagnosis, 6 months later, and yearly thereafter. In order to quantify the degree of visual disturbance, the bilateral visual acuity and fields were evaluated using a visual impairment scale (VIS), according to the guideline of the German Ophthalmological Society. ${ }^{7}$ Using specific tables based on visual acuity and visual field defects, we calculated the VIS scores. The VIS scores ranged from 0 (best) to 100 (worst). If the results of the visual acuity and visual field tests were not available at the time of visit to the outpatient clinic, the subjective visual symptoms of the patients were included.

\section{Radiological Assessment}

MRI was performed yearly after the diagnosis of nonfunctioning PAs in all patients during the first 2 years. Patients with changes in tumor size during the first 2 years underwent MRI every year. MRI was performed every 2 years in patients in whom there was no change in tumor size during the first 2 years. If the patient complained of visual disturbance during follow-up, MRI and ophthalmological assessments were performed immediately, and if there seemed to be a visual disturbance due to tumor, surgery was performed.

Two observers independently verified the MRI scans obtained in all patients. Based on coronal, sagittal, and axial T1- and T2-weighted images of the pituitary fossa at the first and last MRI studies, the following parameters were measured: 1) distance between the optic chiasm and top of the mass (coronal T2-weighted MRI); 2) cavernous sinus invasion (coronal T1-weighted MRI, Knosp Grade 3 
TABLE 1. Baseline characteristics of nonfunctioning PAs according to the initial tumor size

\begin{tabular}{lccrr}
\hline & Total $(\mathrm{n}=197)$ & Microadenoma $(\mathrm{n}=38)$ & Macroadenoma $(\mathrm{n}=159)$ & $p$ Value \\
\hline Mean age, yrs & $53.2 \pm 13.8$ & $43.1 \pm 13.7$ & $55.6 \pm 12.8$ & $<0.001$ \\
\hline Male sex & $96(48.7)$ & $8(21.1)$ & $88(55.4)$ & $<0.001$ \\
\hline Initial tumor vol, $\mathrm{cm}^{3}$ & $2.63 \pm 2.74$ & $0.46 \pm 0.32$ & $3.14 \pm 2.81$ & $<0.001$ \\
\hline Cystic tumor* & $21(10.7)$ & $3(7.9)$ & $18(11.3)$ & 0.771 \\
\hline Distance btwn tumor \& optic chiasm $<1 \mathrm{~mm}$ & $97(49.2)$ & $2(5.3)$ & $95(59.8)$ & $<0.001$ \\
\hline Cavernous sinus invasion & $47(23.9)$ & $0(0)$ & $47(29.6)$ & $<0.001$ \\
\hline Hormone deficiency & $40(20.3)$ & $2(5.3)$ & $38(23.9)$ & 0.012 \\
\hline Median FU duration, yrs $(95 \% \mathrm{Cl})$ & $3.1(1.0-14.9)$ & $3.3(1.0-14.3)$ & $3.0(0.3-14.9)$ & 0.587 \\
\hline
\end{tabular}

$\mathrm{FU}=$ follow up.

Data are presented as mean \pm standard deviation or number (\%) unless otherwise indicated.

${ }^{*}$ Cystic portion $>50 \%$.

or higher); 3) micro- or macroadenoma (coronal, sagittal, and axial T2-weighted MRI); 4) cystic or solid (coronal T2-weighted MRI, cystic portion > 50\%); and 5) volume (coronal T2-weighted coronal MRI).

Tumor volume was calculated by multiplying the tumor area measurement by the contour lines of each coronal view and the slice thickness. In order to reduce measurement errors, the volume was measured twice, and the mean value was used. Meaningful tumor growth was defined as the final volume of the tumor that exceeded $120 \%$ of the initial volume. Meaningful decrease in tumor size was defined as the final volume being less than $80 \%$ of the initial volume. A stable tumor was defined as one that did not increase or decrease in size.

\section{Statistical Analysis}

Data are shown as the number of patients (\%) for categorical variables and mean \pm standard deviation or median (95\% confidence interval [CI]) for continuous variables. A comparison between the 2 groups was performed using the Student t-test for numeric variables and the chi-square test for categorical variables. In the survival analysis, the incidence rates per 100 person-years (PYs) were presented for each variable. MaxStat, a maximal chi-square method in $\mathrm{R}$ version 3.4.0 (R Development Core Team, http:// www.R-project.org), was used to identify the optimal cut points for the rate of pituitary tumor volume growth, predicting the new decline of the visual function. The KaplanMeier survival curve with the log-rank test is presented. All statistical analyses were performed using IBM SPSS software (version 22, IBM Corp.), and differences with a $\mathrm{p}$ value $<0.05$ were considered statistically significant.

\section{Results}

The baseline characteristics of the participants with nonfunctioning PAs are shown in Table 1. Among the 197 patients, macroadenomas $(\mathrm{n}=159,80.7 \%)$ were more prevalent than microadenomas $(\mathrm{n}=38,19.3 \%)$. The mean age of the participants was $53.2 \pm 13.8$ years, and men accounted for $48.7 \%$. The median follow-up duration was 37 months (95\% CI 12-170 months). Patients with macroadenomas were older and were more likely to be male than those with microadenomas. More macroadenomas than microadenomas had a distance between the tumor and the optic chiasm of $<1 \mathrm{~mm}(59.8 \%$ vs $5.3 \%)$ and had cavernous sinus invasion (29.6\% vs $0 \%$ ). However, the prevalence of the cystic tumors and the follow-up durations were similar between the 2 groups.

Figure 1 shows the natural course of the 197 patients with nonfunctioning PAs during the follow-up period. The tumor size increased in 87 (44.2\%) patients and decreased in $27(13.7 \%)$ patients. Visual symptoms and pituitary apoplexy occurred in $5(2.5 \%)$ and $6(3.0 \%)$ patients, respectively. Overall, $8(4.0 \%)$ patients experienced new endocrine dysfunction, and 5 (2.5\%) had an improvement in endocrine function. Among patients with increased tumor sizes, new endocrine dysfunction occurred in 6 (6.9\%), apoplexy in 2, and worsening visual symptoms in 4 patients. However, even in patients with decreased tumor size, new endocrine dysfunction occurred in 1 patient after apoplexy, but improved endocrine dysfunction was observed in 3 patients. Endoscopic transsphenoidal surgery was performed in 8 patients with worsening visual function $(n=5)$ and tumor enlargement $(n=3)$. Worsening visual function improved in the 5 patients after surgery.

We compared the incidences of events (per 100 PYs) in patients with macroadenomas and microadenomas (Table 2). The overall incidences of worsening visual function, surgery during the follow-up period, endocrine dysfunction, and apoplexy were rare (around 0.69-1.11 per 100 PYs). For microadenomas, worsening visual function, surgery, endocrine dysfunction, and apoplexy did not occur, although the difference between the 2 groups was not significant. The incidences of tumor volume growth $\geq 20 \%$ and any growth (tumor volume growth $>0 \%$ ) per $100 \mathrm{PYs}$ were 12.1 (95\% CI 9.78-14.9) and 18.6 (95\% CI 15.7-22.0), respectively. In addition, the incidences of tumor volume growth $\geq 20 \%$ and any growth were significantly higher for macroadenomas than for microadenomas (13.8 vs 4.96 and 20.0 vs 12.8 per 100 PYs, respectively). Figure 2 presents the Kaplan-Meier survival curve for any tumor growth in patients, according to the presence of macroadenomas or microadenomas. The median time to any tumor growth was 4.8 years (95\% CI 3.4-4.8 years) for microadenomas and 4 years (95\% CI 3.3-4.2 years) for macroadenomas.

Using the MaxStat statistical method, we evaluated the cutoff value of the tumor volume growth rate between the 


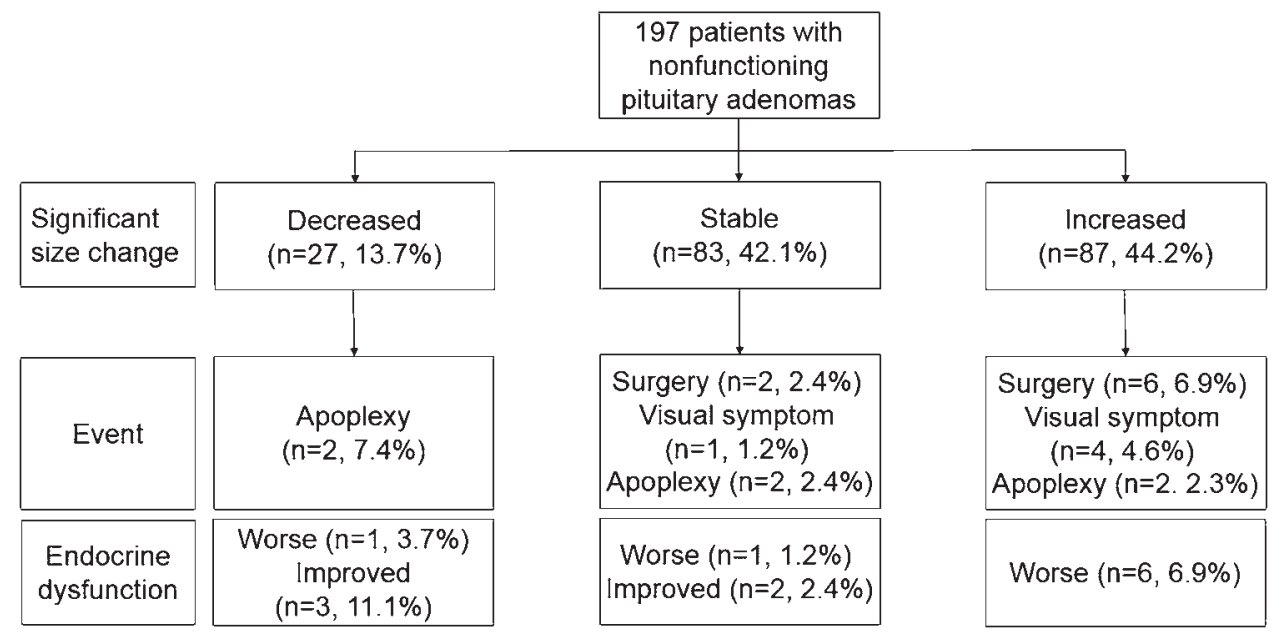

FIG. 1. Natural course of 197 patients with nonfunctioning pituitary adenomas.

first and second MRI studies, which predicted worsening visual function (Table 3). Cases of tumors with a volume growth rate $\geq 0.35 \mathrm{~cm}^{3} /$ year had significantly higher incidences of worsening visual functions than those with a volume growth rate $<0.88 \mathrm{~cm}^{3} /$ year $(4.69$ [95\% CI $1.51-$ $14.5]$ vs 0.30 [95\% CI $0.08-1.21]$ per 100 PYs, $p<0.001$ ).

We further analyzed which parameters affected the incidences of worsening visual function and tumor volume growth $\geq 20 \%$ (Table 4 ). The presence of macroadenomas, distance from the optic chiasm $<1 \mathrm{~mm}$, tumor volume growth $\geq 20 \%$, and any growth were not related to worsening visual function. A tumor volume growth rate $\geq$ $0.88 \mathrm{~cm}^{3} /$ year was the only determinant for worsening visual function. The incidences of tumor enlargement were higher in men than in women. Tumors that were located < $1 \mathrm{~mm}$ away from the optic chiasm were likely to increase in size. Cystic tumors tended to decrease in size spontaneously.

\section{Discussion}

The present study demonstrated that the overall incidence of worsening visual functions was $2.5 \%(n=5 / 197)$ during the median follow-up duration of 37 months, and patients with microadenomas rarely developed worsening visual function, pituitary apoplexy, or endocrine dysfunction. Macroadenomas significantly increased in size compared with microadenomas; however, this growth did not always correlate with worsening visual function. The median time to any tumor growth was 4.8 years $(95 \%$ CI $3.4-4.8$ years) for microadenomas and 4 years $(95 \%$ CI 3.3-4.2 years) for macroadenomas. The cutoff value of $0.88 \mathrm{~cm}^{3} /$ year of the tumor volume growth rate was an important predictor for visual outcomes.

We demonstrated that growth in tumor volume $\geq 20 \%$ was found in $14 \%$ of patients with macroadenomas per year, and $5 \%$ of patients with microadenomas per year. Similarly, Lenders et al. reported that 9 of 23 patients with macroadenomas and 2 of 27 patients with microadenomas developed meaningful tumor growth during a median follow-up period of 36 months. ${ }^{15}$ Any growth (tumor enlargement $>1 \mathrm{~mm}$ ) occurred in $20 \%$ of patients with macroadenomas per year and $13 \%$ of patients with microadenomas per year. A meta-analysis showed that increases in tumor size occurred in 12.5 per $100 \mathrm{PYs}$ for macroadenomas and 3.3 per 100 PYs for microadenomas..$^{10}$ In a recent study on 99 patients with nonfunctioning PAs without surgery, $20 \%$ of the cases showed an increase in size $\geq 2 \mathrm{~mm}$ for approximately 3 years..$^{12}$ Previously conducted studies as well as the present study have shown that cystic tumors have a tendency to decrease in size. ${ }^{10,12}$ The incidence of worsening visual function was 0.65 per 100 PYs in a metaanalysis, which was comparable to our data (0.69 per 100 PYs).${ }^{10}$ The distance between the optic chiasm and tumors

TABLE 2. Incidence of events per 100 PYs in patients with nonfunctioning PAs according to initial tumor size

\begin{tabular}{lcccc}
\hline & \multicolumn{4}{c}{ Incidence/100 PYs $(95 \% \mathrm{Cl})$} \\
\cline { 2 - 5 } & Total & Microadenoma $(\mathrm{n}=38)$ & Macroadenoma $(\mathrm{n}=159)$ & $\mathrm{p}$ Value \\
\hline Worsening visual function & $0.69(0.29-1.66)$ & 0 & $0.87(0.36-2.07)$ & 0.168 \\
\hline Endocrine dysfunction & $1.11(0.55-2.22)$ & 0 & $1.38(0.69-2.76)$ & 0.088 \\
\hline Apoplexy & $0.83(0.37-1.85)$ & 0 & $1.03(0.46-2.30)$ & 0.135 \\
\hline Tumor growth $\geq 20 \%$ & $12.1(9.78-14.9)$ & $4.96(2.36-10.4)$ & $13.8(11.1-17.2)$ & 0.002 \\
\hline Any growth & $18.6(15.7-22.0)$ & $12.8(8.03-20.2)$ & $20.0(16.7-24.0)$ & 0.033 \\
\hline
\end{tabular}

Data are shown as incidence (per 100 person-years) and $95 \%$ confidence interval. 


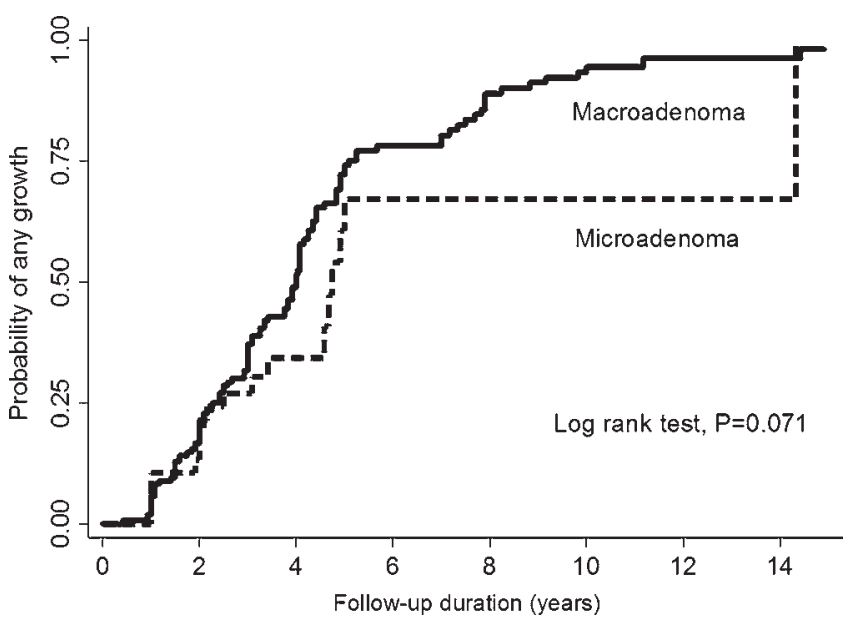

FIG. 2. Kaplan-Meier survival curve for probability of any growth according to initial tumor size.

was not related to worsening visual functions in the present study, and this finding indicates that even PAs near the optic chiasm did not always cause visual field defects. In addition, visual field defects rarely occur even if the PAs enlarged significantly, according to this study. Therefore, it implied that cases of macroadenomas without visual field defects could be managed conservatively, even though it was observed that the mass abutted the optic chiasm, when MRI was performed.

In the first study pertaining to the tumor volume growth rate, Lenders et al. demonstrated that a growth rate $>1.2$ $\mathrm{cm}^{3} /$ year among the macroadenoma group was highly predictive of $\mathrm{a} \geq 20 \%$ increase in volume or surgery. ${ }^{15} \mathrm{We}$ also calculated the tumor volume growth rate between the first and the second MRI studies, rather than tumor volume growth $\geq 20 \%$, to predict worsening of visual function. The cutoff level of $0.88 \mathrm{~cm}^{3} /$ year, which corresponds to $5.9 \mathrm{~mm}$ in tumor diameter, if the tumor was assumed to be a circle, predicted worsening visual function. A metaanalysis, consistent with the present study, reported that an average growth $>3.5 \mathrm{~mm}$ was highly correlated with worsening visual function. ${ }^{10}$

The timing of MRI follow-up for asymptomatic PAs has not been established clearly. Even the Endocrine Society admitted that the imaging follow-up protocol in the guidelines was based on the experience of the task force members. ${ }^{11}$ These guidelines suggest that MRI be repeat- ed half-yearly for 1 year, and then annually for 3 years, in the cases of macroadenomas, and yearly for 3 years in the cases of microadenomas. ${ }^{11}$ On the basis of our data, we suggest that follow-up MRI can be performed at 3 years for microadenomas, whereas the second MRI study for macroadenomas should be done between 6 months and 1 year to assess the tumor growth rate. In patients with a tumor growth rate $\geq 0.88 \mathrm{~cm}^{3} /$ year, MRI should be followed up within 2 years. In cases with tumor growth rate $<0.88$ $\mathrm{cm}^{3} /$ year, the MRI study can be delayed until 4 years. This follow-up period for MRI was longer than that in the proposed protocol by the Endocrine Society.

A recent meta-analysis showed that new hormone deficiency developed in $2.4 \%$ of all patients per year. ${ }^{10} \mathrm{We}$ did not assess the growth hormone axis due to the lack of data. This is why the incidences of endocrine dysfunction in our study (1.1\% per year) were a little lower than in the previous report (2.4\% per year). ${ }^{10}$ None of microadenomas developed endocrine dysfunction, which was consistent with findings in previous studies. ${ }^{2,4,6,8,15,19,20}$ The Endocrine Society's guidelines also did not recommend further follow-up endocrine testing for microadenomas. ${ }^{11}$ However, in the present study, it was found that the rapid growth of PAs ( $\geq 0.88 \mathrm{~cm}^{3} /$ year) was related to hormone deficiency. Likewise, hormone function improved in $11 \%$ of the patients with PAs with decreased sizes. A recent Canadian study also revealed that a change in hormone status was associated with a change in tumor size. ${ }^{12}$

Pituitary apoplexy occurred only in the patients with macroadenomas in the present study (0.83 per 100 PYs). In addition, Sanno et al. reported that pituitary apoplexy occurred in 1 of 248 patients $(0.4 \%) .{ }^{20}$ In a study by Lenders et al. pituitary apoplexy occurred in 1 patient with a microadenoma..$^{15}$ The incidence of pituitary apoplexy was not significantly different between the cases of microadenomas and macroadenomas in the present study due to the rarity of the event, which was also supported by findings in a previous meta-analysis. ${ }^{10} \mathrm{~A}$ rapid growth rate was found to be related to pituitary apoplexy in the meta-analysis, but our study failed to gain significance.

The present study has several strengths. To our knowledge, our study included the largest sample size in a single center. Most early studies were conducted in fewer than 50 patients with nonfunctioning PAs. Sanno et al. published the survey data of 115 conservatively treated nonfunctioning PAs in Japan, and Imran et al. reported the natural history of 99 nonfunctioning PAs among 328 cases of sellar masses in Canada. ${ }^{12,20}$ The definition of an increase in size

TABLE 3. Incidence of events per $100 \mathrm{PYs}$ in patients with nonfunctioning PAs according to tumor volume growth rate

\begin{tabular}{lccr}
\hline & \multicolumn{2}{c}{ Tumor Vol Growth Rate, $\mathrm{cm}^{3} / \mathrm{yr}$} & \multirow{2}{*}{$\mathrm{p}$ Value } \\
\cline { 2 - 3 } & $\geq 0.88(\mathrm{n}=20)$ & $<0.88(\mathrm{n}=177)$ & $<0.001$ \\
\hline Worsening of visual function & $4.69(1.51-14.5)$ & $0.30(0.08-1.21)$ & 0.067 \\
\hline Endocrine dysfunction & $3.13(0.78-12.5)$ & $0.91(0.41-2.01)$ & 0.395 \\
\hline Apoplexy & $1.56(0.22-11.1)$ & $0.75(0.31-1.81)$ & 0.361 \\
\hline Mean FU duration, yrs & $3.8 \pm 2.6$ & $3.2 \pm 1.7$ & \\
\hline
\end{tabular}

Data are shown as incidence $(95 \% \mathrm{Cl})$ except where indicated. 
TABLE 4. Incidence per 100 PYs of worsening visual field and tumor growth $\geq 20 \%$ according to clinical and radiological parameters

\begin{tabular}{|c|c|c|}
\hline & Incidence $(95 \% \mathrm{Cl})$ & $p$ Value \\
\hline Overall worsening visual function & $0.69(0.29-1.66)$ & \\
\hline Microadenoma & 0 & 0.168 \\
\hline Macroadenoma & $0.87(0.36-2.07)$ & \\
\hline Distance from optic chiasm, $\mathrm{mm}$ & & 0.267 \\
\hline$\geq 1$ & $0.50(0.13-2.01)$ & \\
\hline$<1$ & $0.93(0.30-2.87)$ & \\
\hline \multicolumn{3}{|l|}{ Tumor growth, \% } \\
\hline$<20$ & $0.25(0.04-1.81)$ & 0.078 \\
\hline$\geq 20$ & $1.22(0.46-3.24)$ & \\
\hline$\leq 0$ & 0 & 0.077 \\
\hline$>0$ & $1.01(0.42-2.42)$ & \\
\hline Growth rate, $\mathrm{cm}^{3} / \mathrm{yr}$ & & $<0.001$ \\
\hline$<0.88$ & $0.30(0.08-1.21)$ & \\
\hline$\geq 0.88$ & $4.69(1.51-14.5)$ & \\
\hline \multicolumn{3}{|l|}{ Tumor growth $\geq 20 \%$} \\
\hline Overall & $12.06(9.78-14.88)$ & \\
\hline Female & $8.66(6.22-12.07)$ & 0.002 \\
\hline Male & $16.39(12.49-21.50)$ & \\
\hline Microadenoma & $4.96(2.36-10.40)$ & 0.033 \\
\hline Macroadenoma & $13.79(11.08-17.17)$ & \\
\hline Distance from optic chiasm, mm & & 0.003 \\
\hline$\geq 1$ & $8.66(6.22-12.07)$ & \\
\hline$<1$ & $16.39(12.49-21.50)$ & \\
\hline Cystic tumor & $8.69(3.90-19.33)$ & 0.016 \\
\hline Solid tumor & $19.62(16.50-23.34)$ & \\
\hline
\end{tabular}

was either quite variable or not mentioned in most of the studies. ${ }^{2,4,8,18,20}$ However, in the present study, we clearly defined the increase in size as the percentage of tumor volume growth $>20 \%$. The tumor volume measurement was more accurate than the single measure of maximal tumor diameter. There is only one previously conducted study that has measured the tumor volume instead of the diameter. ${ }^{15}$ To elucidate the cutoff values of the tumor volume growth rate for worsening visual function, we used the MaxStat statistical method rather than receiver operating characteristic curve analysis, considering the time variable. Furthermore, to predict worsening visual function, we measured the distance between the optic chiasm and the pituitary tumor and analyzed its effect on visual field defects. We also assessed endocrine function, which was not conducted in the second largest, previously conducted study by Sanno et al. ${ }^{20}$ For comparison with other studies with varied follow-up periods, we presented our data using 100 PYs.

The present study has some limitations. Due to the retrospective cohort design, the hormone tests, visual field examinations, and sella MRI studies were not always conducted under the sample protocol in all patients. However, most of the patients have undergone follow-up annually or biennially. The follow-up period was not long enough to evaluate the outcome of the PAs for more than 10 years. In terms of assessing endocrine function, growth hormone deficiency was not fully assessed. In older men, age-related hypogonadism could not be differentiated from PArelated hypogonadism.

\section{Conclusions}

The present study showed that microadenomas have a benign natural course, which is different from the course of macroadenomas. Microadenomas may not require repeated visual field tests or hormone assessment unless the tumor size increases significantly or new symptoms develop. A tumor volume growth rate $\geq 0.88 \mathrm{~cm}^{3} /$ year was more predictive of worsening visual function and endocrine dysfunction. In patients with microadenomas, the next MRI study can be performed at 3 years. In patients with macroadenomas, we need to follow up the second MRI study between 6 months and 1 year to assess the tumor growth rate. In patients with a tumor growth rate $\geq$ $0.88 \mathrm{~cm}^{3} / y e a r$, the MRI study should be obtained within 2 years. In cases with tumor growth rate $<0.88 \mathrm{~cm}^{3} /$ year, the MRI study can be delayed until 4 years. During the followup period, patients should be educated regarding the visual symptoms and apoplexy symptoms. However, our followup strategy needs to be validated through a long-term prospective cohort study.

\section{Acknowledgments}

We thank all colleagues who contributed to the study. This study was supported by a grant (no. HI16C-1111-020016 to Y. H. Kim) from the Korea Health Industry Development Institute (KHIDI) funded by the Ministry of Health \& Welfare, Republic of Korea, and grants (no. NRF-2017R1A2B2008412 to Y. H. Kim and no. NRF-2017R1D1A1B03031879 to J. H. Kim) from the National Research Foundation of Korea by the Ministry of Science, ICT, and Future Planning (MSIP) of Korea.

\section{References}

1. Agustsson TT, Baldvinsdottir T, Jonasson JG, Olafsdottir E, Steinthorsdottir V, Sigurdsson G, et al: The epidemiology of pituitary adenomas in Iceland, 1955-2012: a nationwide population-based study. Eur J Endocrinol 173:655-664, 2015

2. Arita K, Tominaga A, Sugiyama K, Eguchi K, Iida K, Sumida M, et al: Natural course of incidentally found nonfunctioning pituitary adenoma, with special reference to pituitary apoplexy during follow-up examination. J Neurosurg 104:884-891, 2006

3. Daly AF, Rixhon M, Adam C, Dempegioti A, Tichomirowa MA, Beckers A: High prevalence of pituitary adenomas: a cross-sectional study in the province of Liege, Belgium. J Clin Endocrinol Metab 91:4769-4775, 2006

4. Day PF, Guitelman M, Artese R, Fiszledjer L, Chervin A, Vitale NM, et al: Retrospective multicentric study of pituitary incidentalomas. Pituitary 7:145-148, 2004

5. Dekkers OM, Hammer S, de Keizer RJ, Roelfsema F, Schutte PJ, Smit JW, et al: The natural course of non-functioning pituitary macroadenomas. Eur J Endocrinol 156:217-224, 2007

6. Donovan LE, Corenblum B: The natural history of the pituitary incidentaloma. Arch Intern Med 155:181-183, 1995

7. Fahlbusch R, Schott W: Pterional surgery of meningiomas of the tuberculum sellae and planum sphenoidale: surgical 
results with special consideration of ophthalmological and endocrinological outcomes. J Neurosurg 96:235-243, 2002

8. Feldkamp J, Santen R, Harms E, Aulich A, Mödder U, Scherbaum WA: Incidentally discovered pituitary lesions: high frequency of macroadenomas and hormone-secreting adenomas-results of a prospective study. Clin Endocrinol (Oxf) 51:109-113, 1999

9. Fernandez A, Karavitaki N, Wass JA: Prevalence of pituitary adenomas: a community-based, cross-sectional study in Banbury (Oxfordshire, UK). Clin Endocrinol (Oxf) 72:377-382, 2010

10. Fernández-Balsells MM, Murad MH, Barwise A, GallegosOrozco JF, Paul A, Lane MA, et al: Natural history of nonfunctioning pituitary adenomas and incidentalomas: a systematic review and metaanalysis. J Clin Endocrinol Metab 96:905-912, 2011

11. Freda PU, Beckers AM, Katznelson L, Molitch ME, Montori VM, Post KD, et al: Pituitary incidentaloma: an endocrine society clinical practice guideline. J Clin Endocrinol Metab 96:894-904, 2011

12. Imran SA, Yip CE, Papneja N, Aldahmani K, Mohammad S, Imran F, et al: Analysis and natural history of pituitary incidentalomas. Eur J Endocrinol 175:1-9, 2016

13. Jung KW, Ha J, Lee SH, Won YJ, Yoo H: An updated nationwide epidemiology of primary brain tumors in republic of Korea. Brain Tumor Res Treat 1:16-23, 2013

14. Karamouzis I, Berardelli R, Prencipe N, Berton A, Bona C, Stura G, et al: Retrospective observational analysis of nonirradiated non-functioning pituitary adenomas. J Endocrinol Invest 38:1191-1197, 2015

15. Lenders N, Ikeuchi S, Russell AW, Ho KK, Prins JB, Inder WJ: Longitudinal evaluation of the natural history of conservatively managed nonfunctioning pituitary adenomas. Clin Endocrinol (Oxf) 84:222-228, 2016

16. Molitch ME: Diagnosis and treatment of pituitary adenomas: a review. JAMA 317:516-524, 2017

17. Murad MH, Fernández-Balsells MM, Barwise A, GallegosOrozco JF, Paul A, Lane MA, et al: Outcomes of surgical treatment for nonfunctioning pituitary adenomas: a systematic review and meta-analysis. Clin Endocrinol (Oxf) 73:777-791, 2010

18. Nishizawa S, Ohta S, Yokoyama T, Uemura K: Therapeutic strategy for incidentally found pituitary tumors ("pituitary incidentalomas"). Neurosurgery 43:1344-1350, 1998

19. Reincke M, Allolio B, Saeger W, Menzel J, Winkelmann W: The 'incidentaloma' of the pituitary gland. Is neurosurgery required? JAMA 263:2772-2776, 1990

20. Sanno N, Oyama K, Tahara S, Teramoto A, Kato Y: A survey of pituitary incidentaloma in Japan. Eur J Endocrinol 149:123-127, 2003

21. Tjörnstrand A, Gunnarsson K, Evert M, Holmberg E, Ragnarsson $\mathrm{O}$, Rosén $\mathrm{T}$, et al: The incidence rate of pituitary adenomas in western Sweden for the period 2001-2011. Eur J Endocrinol 171:519-526, 2014

\section{Disclosures}

The authors report no conflict of interest concerning the materials or methods used in this study or the findings specified in this paper.

\section{Author Contributions}

Conception and design: YH Kim, JH Kim, Ji Hyun Lee, Hong, Shin. Acquisition of data: YH Kim, Dho, Jung Hyun Lee. Analysis and interpretation of data: JH Kim, Dho. Drafting the article: JH Kim, Dho. Statistical analysis: JH Kim, Dho. Study supervision: YH Kim.

\section{Correspondence}

Yong Hwy Kim: Seoul National University Hospital, Seoul, Republic of Korea. kimyh96@snu.ac.kr. 